with HPV. Three Socioeconomic status (SES) groups were established (Graffar).

EditorResults 73 SLE patients and 104 healthy control were included. Table 1: Demographics characteristics.

SLE patients 25/73 (34.3\%) had HPV versus 6/104 (5.8\%) in the control group $(p=0.00)$.

In the SLE-HPV were found statistically significant differences in: low SES, sexual partners $\geq 5$, antiDNA+ and low complement and a trend to low educational level $(\mathrm{p}=0,07)$.

At check data the average dose of steroids was $10.8 \mathrm{Mg} / \mathrm{d}$ (SLE-HPV) vs $2.9 \mathrm{Mg} / \mathrm{d}$ in without HPV $(\mathrm{p}=0.00)$ while $61 \%$ (HPV group) vs 29\% (without HPV) were receiving immunosupressors (IS) (Table 2)

Non-differences were found related to duration of SLE, smoking, beginning of sexual intercourse, condom use and anal or oral intercourse.

Conclusions The frequency of HPV was high in women with SLE. We remark oligo/asymptomatic HPV and its association with low SES, serological activity and treatment.

As we detected a high frequency of sole anal lesions we highlight the anoscopy regardless of symptoms.

\section{ASSOCIATION OF SMOKING WITH VASCULAR DAMAGES IN SYSTEMIC LUPUS ERYTHEMATOUS FROM KOREAN LUPUS NETWORK (KORNET) REGISTRY}

L Chan UK*, K Seong-Kyu, C Jung-Yoon, P Sung- Hoon, L Hwajeong, K Ji-Na, K Ji-Won. Catholic University of Daegu School of Medicine, Division of Rheumatology-Department of Internal Medicine, Namgu, Republic of Korea

\subsection{6/lupus-2017-000215.152}

Background and aims To investigate association between smoking and vascular damages in patients with systemic lupus erythematous (SLE).

Methods A total of 500 SLE patients were enrolled in KORean lupus Network (KORNET) registry from January 2014 to January 2016. Disease activity and organ damage were measured by Systemic Lupus Erythematosus Disease Activity Index (SLEDAI) score and Systemic Lupus International Collaborating Clinics (SLICC) damage index. Association analyses using multivariate logistic regression analysis of covariance models with smoking status (three groups: current smoker, ex-smoker, never smoker) as outcome variable were conducted. We were divided into two groups depending on SLICC items (vascular vs. nonvascular involved items) according to the presence or absence of vascular damage including cardiovascular and peripheral vascular systems. Laboratory data was obtained including autoantibodies (antiphospholipid antibodies, anti-double-stranded DNA, etc.), complements, Creactive protein.

Results There are significant differences in vascular component score of SLICC score among current, ex-, and never smokers $(0.17 \pm 0.38,0.03 \pm 0.17$, and $0.03 \pm 0.20, p=0.003)$. whereas overall SLICC scores were similar among three groups $(\mathrm{p}=0.284)$. Current smoker showed higher vascular component score of SLICC score than never smoker $(p=0.014)$ and than ex-smokers $(p=0.039)$. Patients who has history of smoking exposure (current and ex-smoker) showed significantly higher positivity of antiphospholipid antibody (OR 2.58, 95\% CI 1.31-5.08, $\mathrm{p}=0.006$ ).

Conclusions This study revealed that smoking was associated with vascular component scores in SLICC damage index. It suggests that that smoking status may implicate the development of vascular events in SLE.

\section{PREVALENCE AND RISK FACTORS ASSOCIATED WITH NEUTROPENIA IN KOREAN PATIENTS WITH SYSTEMIC LUPUS ERYTHEMATOUS}

${ }^{1}$ M.K. Chung, ${ }^{2}$ IJ Kim, ${ }^{2}$ L Lee*. 'Seoul St. Mary's Hospital- College of Medicine- The Catholic University of Korea, Division of Rheumatology- Department of Internal medicine, Seoul, Republic of Korea; ${ }^{2} E$ wha Womans University School of Medicine, Division of Rheumatology-Department of Internal medicine, Seoul, Republic of Korea

\subsection{6/lupus-2017-000215.153}

Background and aims This study was performed to identify the prevalence and risk factors that are associated with neutropenia in Korean patients with systemic lupus erythematosus (SLE).

Methods A total 160 admissions of 85 SLE patients between 2006 and 2013 were retrospectively reviewed. Neutropenia was defined as absolute neutrophil count (ANC) below 1500/ $\mathrm{mm}^{3}$. Baseline characteristics of the patients were compared between patients who experienced neutropenia and those without. Clinical and serological factors related to neutropenia episode during admission were analysed.

Results Thirty two (37.6\%) patients experienced neutropenia, and neutropenia episode was found in 35 (21.9\%) of admissions. Most of the neutropenia episodes were mild to moderate. Severe neutropenia of ANC $<500 / \mathrm{mm}^{3}$ occurred in $3.1 \%$ of the cases. Patients with neutropenia had higher frequencies of ANA ( 100.0 vs $86.8 \%, \mathrm{p}=0.042)$ and anti-dsDNA (87.5 vs $60.4 \%, p=0.008)$, and satisfied more SLE classification criteria at the time of the diagnosis than those without (4.8 vs 4.1, $\mathrm{p}=0.014)$ Clinical characteristics at admission such as comorbidities, concomitant medications, and SLEDAI were not different between admissions with and without neutropenia. Anaemia, leukopenia, thrombocytopenia and low complement levels were frequently associated with neutropenia. Co-existence of chronic kidney disease (OR, 16.91; 95\% confidence interval (CI), 2.09-136.6; $\mathrm{p}=0.008)$ and Sjögren's syndrome (OR, 6.48; 95\% CI, 1.46-28.66; p=0.014) was associated with increased risk of developing neutropenia.

Conclusions This study demonstrates that most of neutropenia in SLE patients occur as part of hematologic and immunologic abnormalities. SLE patients with renal damage and Sjogren's syndrome should be closely monitored for development of neutropenia.

\section{RISK OF PULMONARY EMBOLISM IN PATIENTS WITH SYSTEMIC LUPUS ERYTHEMATOSUS}

${ }^{1} \mathrm{~F}$ Du*, ${ }^{1} \mathrm{G}$ qiang, ${ }^{1} \mathrm{~B}$ Chunde, ${ }^{2} \mathrm{~L}$ Ben. ${ }^{1}$ Renji Hospital- School of Medicine- Shanghai Jiaotong University, Department of Rheumatology, Shanghai, China; ${ }^{2}$ Yueyang Hospital of Integrated Traditional Chinese and Western Medicine- Shanghai University of Traditional Chinese Medicine, Department of Rheumatology, Shanghai, China

\subsection{6/lupus-2017-000215.154}

Background and aims To study the risk factors of pulmonary embolism (PE) in SLE patients.

Methods 1739 SLE patients admitted to Renji Hospital between 2005 and 2014(0.9\% prevalence) were enrolled. The clinical data (SLEDAI) and lab data (anti-dsDNA antibody, DDimer, antiphospholipid antibody) were analysed. 